\title{
HLA class II alleles with susceptibility of leprosy in the Mexican Mestizo population
}

\author{
Sergio MercadoCeja ${ }^{1}$, Lucia RangelGamboa ${ }^{2}$, MaríaElisa VegaMemije ${ }^{3}$, Angélica OlivoDíaz ${ }^{4}, J_{u l i o}$ Granados $^{5^{*}}$ \\ ${ }^{1}$ Private Practice. Jaime Balmes 11, Torre B, Interior 112-C (Plaza Polanco), Los Morales Polanco11510, Miguel Hidalgo, Mexico, \\ ${ }^{2}$ Department of Ecology of Pathogenic Agents, Research Division, ${ }^{3}$ Department of Dermatology, ${ }^{4}$ Department of Molecular Biology \\ and Histocompatibility, General Hospital "Dr. Manuel Gea Gonzalez", Mexico City 14080, Mexico, Department of Transplantation, \\ Instituto Nacional de Ciencias Médicas y Nutrición Salvador Zubirán, Mexico City 14080, Mexico.
}

\begin{abstract}
Leprosy is a chronic, infectious disease, caused by Mycobacterium leprae, Mycobacterium lepromatosis or both, which affects the peripheral nervous system and the skin. Activation of cellular immunity in infected individuals depends on antigen recognition, which involves relevant HLA-Class II alleles. Therefore, the objective of this study was to determine HLA-Class II allele frequencies (HLA-DRBland-DQB1)in Mexican Mestizo leprosy patients and compare themwith healthy controls, in order to define their role in the genetic susceptibility to this infection. The genomic DNA of each participant was obtained from peripheral blood, using the salting-out method. PCR amplification and hybridization ofHLA-class II alleleswas made by PCR-SSO. The results showed that frequencies of HLA-DRB $1 * 15(P c=0.003, \mathrm{OR}=3.395 \% \mathrm{CI}=1.53-7.33), \mathrm{HLA}-\mathrm{DQB}{ }^{*} 05(P c$ $=0.00003, \mathrm{OR}=6.0395 \% \mathrm{CI}=2.49-14.61)$ and HLA-DQB $1 * 06(P c=0.007, \mathrm{OR}=2.89,95 \% \mathrm{CI}=1.38-6.04)$ were significantly higher among leprosypatients than those of healthy controls. The study suggests that HLADRB1*15, HLA-DQB1*05, and HLA-DQB1*06 are associated with leprosy susceptibility in the Mexican Mestizo population.
\end{abstract}

Keywords: leprosy, HLA-DR, HLA-DQ, HLA-DRB1*15, HLA-DQB1*05, HLA-DQB1*06

\section{INTRODUCTION}

Despite the World Health Organization strategy for leprosy control, new cases of leprosy continue to emerge across developing areas, such Asia and Latin America, with 119055 and 31527 registered cases by the end of 2017, representing a prevalence of 0.6 and 0.31 per 10000 inhabitants, respectively ${ }^{[1-2]}$. In 2017,
Mexico reported more than 100 new cases (140 cases), which is considered a high rate of diagnoses ${ }^{[3]}$.

Leprosy is a chronic infectious disease that affects genetically predisposed individuals. It is produced by Mycobacterium leprae (M. leprae), Mycobacterium lepromatosis (M. lepromatosis) or both, which are acid-fast bacilli ${ }^{[4]}$. Clinical infection with these Mycobacteria are mainly characterized by affecting the

\footnotetext{
*Correspondence to: Lucia Rangel-Gamboa, Department of Ecology of Pathogenic Agents, Research Division, General Hospital "Dr. Manuel Gea González". Calzada de Tlalpan 4800, Tlalpan Centro I, Tlalpan, Ciudad de México, México. C.P 14080. Tel: 0052 40003000 ext. 6100 , E-mail: draluciaragel@yahoo.com.mx; Julio Granados: Department of Transplantation, Immunogenetics, Instituto Nacional de Ciencias Médicas y de la Nutrición, Salvador Zubirán. 7a Cerrada de Fray Pedro de Gante 50, Tlalpan, Sección XVI, 14080 Ciudad de México, México.Tel: 00 52 55 5485-0080, E-mail: julgrate@yahoo.com. Conflict of interests:No conflicts of interesthave been reported. In addition, we confirm that none of the authors have any professional or financial relationship with the individuals who have been recommended to review themanuscript.
} 
peripheral nerves and the skin. Leprosy is also considered an immunological disease, so the failure of diagnosis and/ or treatment will eventually increase neural disease ${ }^{[5]}$. The clinical spectrum of leprosy includes two poles: (1) benign or tuberculoid leprosy, classified as paucibacillary $(\mathrm{PB}$, bacillary index $\leqslant 2)$, characterized by an efficient cellular immune response, demonstrated by a positive intradermal reaction to lepromin; (2) malignant or lepromatous leprosy(LL), including patients who have a $\mathrm{BI} \geqslant 2$, is classified as multibacillary $(\mathrm{MB})$, which is characterized by a failure of the cellular immune response and impossibility of destruction of the bacillus by the macrophage. Therefore, differences in immune responses to these mycobacterial infections, as well as the innate resistance presented by most people, suggest the involvement of susceptibility genes. Recently, chromosome 6 p21 has been identified as a locus of susceptibility to leprosy in a genome-wide $\operatorname{scan}^{[6]}$. In this chromosome region are founded the human leukocyte antigen (HLA) genes, which have been investigated for their roles in the pathogenesis of leprosy ${ }^{[7]}$. Therefore, according to the previous reports about the association of HLA with leprosy, it is considered that the HLA alleles play a role in the process of antigen presentation and activation of the cellular immune response against intracellular pathogens. Previous reports in northern mexico, have shown genetic susceptibility to leprosy associated with HLA-DR alleles, particularly the HLA-DRB1*15:01 molecular subtype. There is also an association with genetic polymorphisms of certain inflammatory cytokines, namely TNF-alpha and IL- $17^{[8-9]}$.

On the other hand, Mexicans Mestizos are a very heterogeneous group from the genetic standpoint, with a genetic admixture of more than $56 \%$ autochthonous Amerindian genes, around 40\% European genes, and $4 \%$ African genes. Additionally, in Mexico,the incidence of lepromatous leprosy is higher than that of tuberculoid leprosy ${ }^{[10-11]}$. Thus, the objective of this study was to determine HLA-Class II allele frequencies(HLA-DRB1 and-DQB1) in Mexican Mestizo leprosy patients and compare them with healthy controls, in order to define their role in the genetic susceptibility to leprosy.

\section{MATERIALS AND METHODS}

\section{Subjects}

In this study, 42 unrelated Mexican individuals diagnosed for leprosy and registered in the "National Program for the Control of Leprosy" plus 99 control samples were included. The patients were additionally classified as multibacillary (MB) or paucibacillary (PB) based on clinical and histological criteria. Ethically, patients and controls were classified as Mestizos: individuals born in Mexico, with Mexican ancestors going back at least three generations. Mestizos are the consequence of 500 years of admixture between Amerindians (Asian origin), Spaniards and Africans, and represent most Mexican residents ${ }^{[10-11]}$.The control group was composed of ethnically similar healthy individuals from the general population. Sample collection was performed inthe "Dr. Manuel Gea González" General Hospital, the Dermatologic Center of the "Dr. Fernando Latapi", the Dermatologic Hospital of "Dr. Pedro López," and the Control Leprosy Program in San Luis Potosí State over a period of two years. The study was approved by the Ethics and Research Committees of each Institution. Written informed consent was obtained from all subjects before their admission in this study, according to the Helsinki Declaration.

\section{HLA-DRB1 and DQB1 typing}

Genomic DNA from all subjects included in this study were purified from $10 \mathrm{~mL}$ of peripheral blood leukocytes, according to the salting-out method described by Miller ${ }^{[12]}$. The HLA-DRB1 and HLADQB1loci were genotyped based on the hybridization of labeled single-stranded polymerase chain reaction (PCR) products to sequence-specific oligonucleotides (SSO) probes, using the LIFECODES HLA-DRB1 and HLA-DQB1 typing kits(Gen-Probe Immucor, Stanford, CT, USA) and the Luminex platform (Luminex Corp., USA). The Luminex method includes PCR amplification, hybridization, a streptavidinphycoerythrin (SA-PE) reaction, and analytical measurements. Target DNA was amplified by PCR using 5 ' biotin-labeled primers that were highly specific to particular sequences of HLA-DRB1 genes. PCR was carried out in a $20 \mu \mathrm{L}$ reaction containing Lifecodes mixture $(6 \mu \mathrm{L})$, Taq polymerase $(0.2 \mu \mathrm{L})$, nucleasefree water $(11.8 \mu \mathrm{L})$ and genomic DNA $(4 \mu \mathrm{L})$. After denaturization, amplified DNA could hybridize to complementary DNA probes coupled to microbeads and the oligobead-coupled, hybridized PCR product was labeled with streptavidin-phycoerythrin. Finally,HLA alleles were determined by the Quick type for Lifecodes (v3.0) software.

\section{Mitsuda testing}

To determine the cellular immune response of patients and controls, they were injected intradermally with $0.1 \mathrm{~mL}$ of lepromin, containing $4 \times 10^{6}$ cells of $M$. leprae (derived from humans), on the volar surface of the forearm (Mitsuda Lepromin, WHO standard). The 
test was considered positive when the skin induration was equal to or greater than $5 \mathrm{~mm}$ between 24 and 48-hours post-inoculation ${ }^{[13]}$.

\section{Statistical analysis}

Allele frequencies were calculated by direct counting. Differences in the frequencies of HLA class II alleles were analyzed using $\chi^{2}$, and $P$-values less than 0.05 were considered statistically significant. The nominal $P$-values were corrected for multiple testing $(P c$-value) using the Bonferroni correction for allele frequencies (by multiplying the original $P$-value by the of HLA alleles being tested). A $P c$-value of less than 0.05 was accepted as statistically significant. Odds ratios and 95\% confidence intervals (95\% CI) were calculated to measure association strength with Epi Info ${ }^{\mathrm{TM}}$ v7.2 software from CDC (Center for Disease Control and Prevention).

\section{RESULTS}

Forty-two patients passed theinclusion criteria to take part in this study, and were aged between 41 and
90 years old, of which 22 were female, and 20 were male. They were born in the following cities: Coahuila, Guanajuato, Jalisco, Mexico City, Michoacán, Oaxaca, San Luis Potosí, Veracruz, Yucatan, and Zacatecas. According to their clinical history, histopathology studies and the Mitsuda test, most patients were classified as lepromatous leprosy $(n=35,83.3 \%)$, while 4 patients corresponded to the tuberculoid pole $(9.5 \%)$ and 3 patients hadinterpolar cases or dimorphic leprosy $(7.2 \%)$.

The allele frequencies of HLA-DRB $1 *$ alleles in the leprosy patients and control group were summarized in Table 1 and the results of association were analyzed. The frequency HLA-DRB $1 * 15$ allele among patients was 0.19 , which was significantly higher than the frequency of 0.06 observed in healthy controls $(P c$ $=0.003, \mathrm{OR}=3.395 \% \mathrm{CI}=1.53-7.33)$. In a similar way, the allele frequencies of HLA-DRQ1* alleles were presented in Table 2, and exposed a significant association with HLA-DQB1 $* 05(\mathrm{Pc}=0.00003, \mathrm{OR}=6.03$ $95 \% \mathrm{CI}=2.49-14.61)$ and HLA-DQB1 $* 06(\mathrm{Pc}=0.007$, $\mathrm{OR}=2.89,95 \% \mathrm{CI}=1.38-6.04)$.

Table 1 HLA-DRB1 allele frequencies in leprosy patients and healthy controls from Mexico

\begin{tabular}{|c|c|c|c|c|c|c|c|}
\hline \multirow{2}{*}{ Alleles } & \multicolumn{2}{|c|}{ Leprosy patients $(N=84)$} & \multicolumn{2}{|c|}{ Healthy individuals $(N=198)$} & \multirow{2}{*}{$\mathrm{Pc}$} & \multirow{2}{*}{ OR } & \multirow{2}{*}{$95 \% \mathrm{CI}$} \\
\hline & $n$ & gf & $n$ & gf & & & \\
\hline $\mathrm{DRB} 1 * 04$ & 19 & 0.226 & 47 & 0.237 & 0.961 & 0.9 & $0.51-1.72$ \\
\hline DRB1*15 & 16 & 0.190 & 13 & 0.066 & 0.003 & 3.3 & $1.53-7.33$ \\
\hline $\mathrm{DRB} 1 * 08$ & 11 & 0.130 & 33 & 0.167 & 0.564 & 0.8 & $0.36-1.57$ \\
\hline $\mathrm{DRB} 1 * 14$ & 9 & 0.107 & 21 & 0.106 & 1.000 & 1.0 & $0.44-2.31$ \\
\hline $\mathrm{DRB} 1 * 13$ & 7 & 0.083 & 10 & 0.051 & 0.432 & 1.7 & $0.63-4.65$ \\
\hline $\mathrm{DRB} 1 * 03$ & 6 & 0.071 & 11 & 0.056 & 0.811 & 1.3 & $0.47-3.66$ \\
\hline $\mathrm{DRB} 1 * 01$ & 5 & 0.059 & 10 & 0.051 & 0.985 & 1.2 & $0.39-3.59$ \\
\hline $\mathrm{DRB} 1 * 07$ & 5 & 0.059 & 22 & 0.111 & 0.261 & 0.5 & $0.19-1.39$ \\
\hline $\mathrm{DRB} 1 * 09$ & 2 & 0.023 & 3 & 0.015 & 0.992 & 1.6 & $0.26-9.67$ \\
\hline $\mathrm{DRB} 1 * 10$ & 2 & 0.023 & 1 & 0.005 & 0.442 & 4.8 & $0.43-53.73$ \\
\hline $\mathrm{DRB} 1 * 11$ & 1 & 0.011 & 20 & 0.101 & 0.018 & 0.1 & $0.01-0.81$ \\
\hline $\mathrm{DRB} 1 * 16$ & 1 & 0.011 & 5 & 0.025 & 0.795 & 0.5 & $0.04-4.04$ \\
\hline
\end{tabular}

Table 2 HLA-DQB1 allele frequencies in leprosy patients and healthy controls from Mexico

\begin{tabular}{|c|c|c|c|c|c|c|c|}
\hline \multirow{2}{*}{ Alleles } & \multicolumn{2}{|c|}{ Leprosy patients $(N=84)$} & \multicolumn{2}{|c|}{ Healthy individuals $(N=198)$} & \multirow{2}{*}{$\mathrm{Pc}$} & \multirow{2}{*}{ OR } & \multirow{2}{*}{$95 \% \mathrm{CI}$} \\
\hline & $n$ & gf & $n$ & gf & & & \\
\hline $\mathrm{DQB1} * 03$ & 25 & 0.298 & 72 & 0.459 & 0.352 & 0.74 & $0.43-1.29$ \\
\hline DQB1*05 & 17 & 0.202 & 8 & 0.051 & 0.00003 & 6.03 & 2.49-14.61 \\
\hline DQB1*06 & 17 & 0.202 & 16 & 0.102 & 0.007 & 2.89 & $1.38-6.04$ \\
\hline DQB1*04 & 13 & 0.155 & 30 & 0.191 & 1.000 & 1.03 & $0.51-2.08$ \\
\hline DQB1*02 & 12 & 0.143 & 31 & 0.197 & 0.911 & 0.90 & $0.44-1.85$ \\
\hline Other alleles & 0 & 0 & 41 & 0.261 & & & \\
\hline
\end{tabular}

\section{DISCUSSION}

It is well known that only a small percentage of people that have contact with M.lepraeor M. lepromatosis, present with the clinical disease (1\%-3\%), a remark that supports the hypothesis of host genetic factors being involved in the development of leprosy.
Also, previous work reported a male-to-female patient ratio of 2:1, and introduced the possibility of gender association in leprosy ${ }^{[14]}$. Nevertheless, our study did not show the differences in prevalence according to gender. Concerning HLA alleles, we found HLADRBI*15 associated significantly with leprosy compared to healthy individuals, suggesting that HLA- 
DRB $1 * 15$ was involved in genetic susceptibility to leprosy in Mexican Mestizos, as has been previously described in the Han population, in China ${ }^{[7]}$. In previous Mexican work, where only native residents of the state of Sinaloa were included ${ }^{[15]}$, the frequency of HLA-DRB1*15 was almost twice that of healthy individuals, but there was no statistical difference. In this research, HLA-DQB1 alleles, specifically HLADQB $1 * 05$ and HLA-DQB1*06, were found significantly associated with leprosy for the first time. These results agreed with a recent publication that reported the association between HLA-DQB1*05:02 and disseminated nontuberculous mycobacterial infections, finding also that HLA-DQB1*06:01 and HLADQB1*06:09 genes were related to higher susceptibility to pulmonary tuberculosis ${ }^{[16-17]}$. Therefore, these results above suggest that HLA-DQB $1 * 05$ and HLADQB $1 * 06$ alleles are related to susceptibility to mycobacterial infections in general.

On the other hand, Escamilla-Tilch et al. ${ }^{[8]}$, reported an association of HLA-DRB $1 * 01$ with lepromatous and dimorphic leprosy, but with most patients coming from the north-west part of Mexico. In light of this, we considered that place of birth was likely to be related to HLA association with leprosy in Mexico, since the Mexican genetic background differs according to geographical region. The northern population has more European genetic ancestry, while the center and the southern populations contain more Amerindian genes. Consequently, a disparity in HLA-DR and HLA-DQ allele frequencies has been reported in the Mexican population, which may explain the variance in the results between the previous and the present studies $^{[10-11]}$. In relation to the etiological agent, it is important to highlight that the dominant presence of $M$. lepromatosishas been reported in the state of Sinaloa, and considering that the $M$. lepromatosis genome differs in more than $13 \%$ when compared to the M. leprae genome, the differences in the etiological agent mayalso influence the HLA association observed in this work. Also,M. lepromatosis has been previously associated with lepromatous leprosy, especially the dimorphic form (DLL). And, since this clinical presentation predominated in the western states of Mexico, M. Lepromatosis was mostly reported ther $\mathrm{e}^{[17-19]}$. In contrast, our study group included 26 patients from central states, 8 from the western pacific cost, 6 from Caribbean cost, and 2 from the northern state. Thirtyfive patients presented with lepromatous leprosy, nevertheless only 3 had dimorphic forms and only 4 presented with tuberculoid leprosy.

In conclusion, the different HLA-DRB1 associations found in various studies could be related to the genetic background of the specific population and the dominant etiological agent in each region. Thus, HLA-DR alleles are associated with susceptibility to leprosy per se. However, leprosy causal agent species are not well known in each region, and consequently, further studies are required to elucidate the HLA alleles of susceptibility and protection associated with each one of the causative species of leprosy and the possible relation with different genetic ancestries in each population. Whereas, HLA-DQB1 $* 05$ and HLADQB $1 * 06$ alleles could be related to susceptibility to mycobacterial infections in general.

\section{Acknowledgments}

We are grateful tothe people at Nacional Program to Lepra Control, Dermatologic Center "Dr. Fernando Latapi," Dermatologic Hospital "Dr. Pedro López" and Control Lepra Program from San Luis Potosí Estate, who helped us recruitthepatients.

\section{References}

[1] World Health Organization. Global leprosy update, 2015: time for action, accountability, and inclusion[J]. Weekly Epidemiol Rec, 2016, 91(35): 405-420

[2] World Health Organization. Global leprosy update, 2017: reducing the disease burden due to leprosy[J]. Weekly Epidemiol Rec,2018, 93(35): 445-456.

[3] Secretaria de Salud, 2018. Lepra: una enfermedad infecciosa. https://www.gob.mx/salud/articulos/lepra-unaenfermedad-infecciosa

[4] Han XY, Seo YH, Sizer KC, et al. A new Mycobacterium species causing diffuse lepromatous leprosy[J]. Am J ClinPathol, 2008, 130(6):856-864.

[5] Lastória JC, Abreu MA. Leprosy: a review of laboratory and therapeutic aspects-part 2[J]. An Bras Dermatol, 2014, 89(3):389-401

[6] Miller EN, Jamieson SE, Joberty C, et al. Genome-wide scans for leprosy and tuberculosis susceptibility genes in Brazilians[J]. Genes Immun, 2004, 5(1):63-67.

[7] Zhang F, Liu H, Chen S, et al. Evidence for an association of HLA-DRB1*15 and DRB1*09 with leprosy and the impact of DRB1*09 on disease onset in a Chinese Han population[J]. BMC Med Genet, 2009, 11(10):133

[8] Escamilla-Tilch M, Torre-Carrillo NM, Payan RR, et al. Association of genetic polymorphism of HLA-DRB1 antigens with the susceptibility to lepromatous leprosy[J]. Biomed Rep, 2013, 1(6):945-949.

[9] Lugo-Zamudio GE, Barbora-Cobos RE, Delgado-Ochoa D,et al. Genetic markers in HLA region to identify patients with tuberculosis risk, in RA subjects[J]. Int J Clin Rheumatol, 2019, 14(1):16-21.

[10] Rubi-Castellanos R, Anaya-Palafox M, Mena-Rojas E, et al. Genetic data of 15 autosomal STRs (Identifiler kit) 
of three Mexican Mestizo population samples from the States of Jalisco (West), Puebla (Center), and Yucatan (Southeast) [J]. Forensic SciInt Genet, 2009, 3(3):71-76.

[11] Barquera R, Zuñiga J, Hernandez-Díaz R, et al. HLA class I and class $\mathbb{I}$ haplotypes in admixed families from several regions of Mexico[J]. Mol Immunol, 2008, 45(4):11711178.

[12] Miller SA, Dykes DD, Polesky HF. A simple salting out procedure for extracting DNA from human nucleated cells[J]. Nucleic Acids Res, 1988, 16(3):1215.

[13] Roberts PP, Dockrell HM, McAdam PWJ. Evidence that the Mitsuda reaction to Mycobacterium leprae can be mediated by lymphocytes responsive to Mycobacterium tuberculosis[J]. Clin Exp Immunol, 1988, 72(3):390-393.

[14] Peters ES, Eshier EL. Male-female (sex) differences in leprosy patients in South Eastern Nigeria: female present late for diagnosis and treatment and have higher rates of deformity[J]. Lepr Rev, 2002, 73:262-267.

[15] Aguilar-Medina M, Escamilla-Tilch M, Frias-Castro LO,et al. HLA alleles are genetic markers for suscepti- bility and resistance towards leprosy in a Mexican Mestizo population[J]. Ann Hum Genet, 2017, 81(1):35-40.

[16] Chi CY, Chu CC, Liu JP,et al. Anti-IFN- $\gamma$ autoantibodies in adults with disseminated nontuberculous mycobacterial infections are associated with HLA-DRB1*16:02 and HLA-DQB1*05:02 and the reactivation of latent varicella-zoster virus infection[J]. Blood, 2013, 121(8):13571366.

[17] Oliveira-Cortez A, Melo AC, Chaves VE, et al. Do HLA class II genes protect against pulmonary tuberculosis? A systematic review and meta-analysis[J]. Eur J Clin Microbiol Infect Dis, $2016,35(10): 1567-1580$

[18] Han XY, Sizer KC, Velarde-Félix JS, et al. The leprosy agents Mycobacterium lepromatosis and Mycobacterium leprae in Mexico[J]. Int J Dermatol, 2012, 51(8):952-959.

[19] Han XY, Quintanilla M. Diffuse lepromatous leprosy due to Mycobacterium lepromatosis in Quintana Roo, Mexico[J]. J Clin Microbiol, 2015, 53(11):3695-3698

(Received 11 July 2019, Revised 9 August 2019, Accepted 19 August 2019) 